\title{
Microbial degradation of furanic compounds: biochemistry, genetics, and impact
}

\author{
Nick Wierckx • Frank Koopman • \\ Harald J. Ruijssenaars • Johannes H. de Winde
}

Received: 21 July 2011 /Revised: 29 September 2011 /Accepted: 6 October 2011 / Published online: 28 October 2011

(C) The Author(s) 2011. This article is published with open access at Springerlink.com

\begin{abstract}
Microbial metabolism of furanic compounds, especially furfural and 5-hydroxymethylfurfural (HMF), is rapidly gaining interest in the scientific community. This interest can largely be attributed to the occurrence of toxic furanic aldehydes in lignocellulosic hydrolysates. However, these compounds are also widespread in nature and in human processed foods, and are produced in industry. Although several microorganisms are known to degrade furanic compounds, the variety of species is limited mostly to Gram-negative aerobic bacteria, with a few notable exceptions. Furanic aldehydes are highly toxic to microorganisms, which have evolved a wide variety of defense mechanisms, such as the oxidation and/or reduction to the furanic alcohol and acid forms. These oxidation/reduction reactions constitute the initial steps of the biological pathways for furfural and HMF degradation. Furfural degradation proceeds via 2 -furoic acid, which is metabo-
\end{abstract}

$\underline{\text { Nick Wierckx and Frank Koopman contributed equally to this work. }}$

N. Wierckx $\cdot$ H. J. Ruijssenaars

BIRD Engineering BV,

Westfrankelandijk 1,

3115 HG Schiedam, The Netherlands

F. Koopman · J. H. de Winde $(\bowtie)$

Department of Biotechnology, Delft University of Technology and Kluyver Centre for Genomics of Industrial Fermentation, Julianalaan 67 ,

2628 BC Delft, The Netherlands

e-mail: J.H.deWinde@tudelft.nl

Present Address:

N. Wierckx

Institute of Applied Microbiology, RWTH Aachen University,

Worringerweg 1 ,

52074 Aachen, Germany lized to the primary intermediate 2-oxoglutarate. HMF is converted, via 2,5-furandicarboxylic acid, into 2-furoic acid. The enzymes in these HMF/furfural degradation pathways are encoded by eight $h m f$ genes, organized in two distinct clusters in Cupriavidus basilensis HMF14. The organization of the five genes of the furfural degradation cluster is highly conserved among microorganisms capable of degrading furfural, while the three genes constituting the initial HMF degradation route are organized in a highly diverse manner. The genetic and biochemical characterization of the microbial metabolism of furanic compounds holds great promises for industrial applications such as the biodetoxifcation of lignocellulosic hydrolysates and the production of value-added compounds such as 2,5-furandicarboxylic acid.

Keywords Furfural $\cdot$ Hydroxymethylfurfural . Lignocellulosic hydrolysate - Metabolic pathway · Detoxification

\section{Introduction}

Due to the indiscriminate use of fossil resources, the need for renewable feedstocks is becoming increasingly evident. Lignocellulosic biomass is a readily available and abundant feedstock, which can be converted into fermentable sugar by means of thermochemical and/or enzymatic treatments (Taherzadeh et al. 1997; Palmqvist and Hahn-Hagerdal 2000a, b; Lin and Tanaka 2006; Abril and Abril 2009; Parawira and Tekere 2011). These lignocellulosic hydrolysates have been heralded as the future feedstock for fermentative production of biobased fuels and chemicals (Ragauskas et al. 2006). 
For efficient release of sugars from lignocellulosic material, pretreatment of the raw biomass is essential. The pretreatment makes the biomass accessible to hydrolyzing chemicals or enzymes by breaking up the lignin structure and disrupting the crystalline structure of the cellulose fibers (Taherzadeh and Karimi 2008). Although many pretreatment approaches have been developed over the years, dilute acid hydrolysis appears to be the most preferred technique for large-scale application. This pretreatment is relatively harsh, however, giving rise to the formation of unwanted and often inhibitory byproducts such as furanic aldehydes, weak acids, and phenolic compounds (Palmqvist and Hahn-Hagerdal 2000b; Thomsen et al. 2009; Klinke et al. 2004; Larsson et al. 1999).

Of the inhibitors formed during acid pretreatment, the furanic aldehydes are considered particularly undesirable due to their relative abundance and toxic effect (Heer and Sauer 2008; Zaldivar et al. 1999). These furanic aldehydes inhibit the hydrolytic enzymes used to liberate the sugars from the (hemi-)cellulose fractions (Jing et al. 2009), as well as growth and metabolism of the microorganisms used in the subsequent fermentation process (Klinke et al. 2004; Palmqvist and Hahn-Hagerdal 2000b). Thus, the occurrence of compounds like furfural and 5-hydroxymethylfurfural (HMF; Fig. 1) can considerably decrease the yield and productivity of fermentative processes based on lignocellulosic hydrolysate (for reviews, see Almeida et al. 2009; Palmqvist and Hahn-Hagerdal 2000b; Thomsen et al. 2009).

The formation of inhibitors during biomass (pre-)treatment may be prevented by careful control of the process parameters. Although considerable progress has been made in lab-scale hydrolysis processes (Kumar et al. 2009), it should be noted that the formation of inhibitory by-products is not easily prevented in an economical way at an industrial scale. Hence, it is often preferred to remove inhibitors prior to fermentation. Several approaches have been investigated, ranging from overliming to solvent extraction and biological detoxification (Mussatto and Roberto 2004; Palmqvist and Hahn-Hagerdal 2000a; Nichols et al. 2010). Alternatively, microbial fermentation hosts may be selected or engineered to tolerate or even metabolize the toxic compounds from lignocellulose hydrolysates. This approach has resulted in host organisms that can tolerate higher concentrations of furfural (Petersson et al. 2006; Heer and Sauer 2008), that metabolize acetate (Medina et al. 2010), furfural, and HMF (Koopman et al. 2010b; Lopez et al. 2004; Table 1), and that efficiently produce polyhydroxyalkanoates from bagasse hydrolysate (Yu and Stahl 2008). In order to introduce or optimize such properties in a targeted manner, detailed insight into inhibitor tolerance and metabolism is the key. The latter has been the subject of intensive study over the last decade. This review aims at providing an overview of these studies, with a special focus on microbial metabolism of furfural and HMF.

\section{Formation and occurrence of furanic compounds}

The recent academic and industrial interest in furfural and HMF can be attributed to a large extent to their occurrence in lignocellulosic hydrolysates. Up to $7.2 \mathrm{~g} / \mathrm{l}$ of furanic compounds can be found in these hydrolysates, although the exact amount largely depends on the type of lignocellulose used and the pretreatment and hydrolysis process employed (Almeida et al. 2009). However, these compounds can also be detected in other sources. In nature, furfural (derived from Latin furfur, bran) is the most abundant of the simple furanic compounds (Dean 1963). Typically, HMF and furfural are associated with sugar-rich or desiccated foods like honey, coffee, and dried fruits (National Toxicology Program 1990, 2010). Through these foods, humans are regularly exposed to furfural and HMF, as is also evident from the common occurrence of furanic compound derivatives such as 2,5-furandicarboxylic acid in human urine (Flaschenträger and Wahhab 1960). Although there is some evidence of carcinogenic effects from high doses of these furanic aldehydes (National Toxicology Program 1990, 2010), HMF is also explored for its beneficial potential in treating human diseases (Lin et al. 2008; Ding et al. 2010; Michail et al. 2007).

At present, it is unclear whether naturally occurring furanic aldehydes emerge purely from abiotic dehydration or that also enzymatic reactions are involved. Furfural and HMF are formed abiotically by threefold dehydration of pentose, respectively, hexose sugars, usually catalyzed by mineral acid (Chheda et al. 2007). Several publications report on HMF and furfural as fungal metabolites (Sumiki 1931; Kawarada et al. 1954). However, it was not unequivocally demonstrated that their formation was biogenic as their presence may also be attributed to the heat sterilization of growth media or the sample extraction procedure (Dean 1963).

In industry, furfural is chemically produced at large scale for application as solvent or as a building block for resins. HMF and furfural are additionally applied as flavor compounds and in the manufacture of pharmaceuticals (Lin et al. 2008; Ding et al. 2010; Michail et al. 2007).

\section{Furanic compound-degrading microorganisms}

In 1964, Kakinuma and Yamatodani (1964) were the first to report the isolation of microorganisms capable of degrading furanic compounds "for the purpose of demonstrating the metabolism of furan compounds by microorganisms." 
Table 1 Overview of species capable of degrading furanic compounds

\begin{tabular}{|c|c|c|c|c|}
\hline Strain & Year & Substrate & Order $^{\mathrm{a}}$ & Reference \\
\hline Amorphotheca resinae $\mathrm{ZN} 1$ & 2010 & Furfural and $\mathrm{HMF}$ & F Leotiomycetes incertae sedis & Zhang et al. (2010) \\
\hline Cupriavidus basilensis HMF14 & 2010 & Furfural and HMF & - Burkholderiales & Wierckx et al. (2010) \\
\hline Arthrobacter nicotianae & 2010 & Furfural and $\mathrm{HMF}$ & + Actinomycetales & Wierckx et al. (2010) \\
\hline Telluria mixta & 2010 & Furfural and HMF & - Burkholderiales & Wierckx et al. (2010) \\
\hline Burkholderia phytofirmans PsJN & 2010 & Furfural and HMF & - Burkholderiales & Koopman et al. (2010b) \\
\hline Burkholderial phymatum STM815 & 2010 & Furfural and HMF & - Burkholderiales & Koopman et al. (2010b) \\
\hline Bradyrhizobium japonicum USDA110 & 2010 & Furfural and HMF & - Rhizobiales & Koopman et al. (2010b) \\
\hline Rhodopseudomonas palustris BisB18 & 2010 & Furfural & - Rhizobiales & Koopman et al. (2010b) \\
\hline $\begin{array}{l}\text { Methylobacterium radiotolerans } \\
\text { JCM2831 }\end{array}$ & 2010 & Furfural and HMF & - Rhizobiales & Koopman et al. (2010b) \\
\hline Dinoroseobacter shibae DFL 12 & 2010 & Furfural & - Rhodobacterales & Koopman et al. (2010b) \\
\hline Ralstonia eutropha $\mathrm{H} 16$ & 2010 & Furfural & - Burkholderiales & Koopman et al. (2010b) \\
\hline Burkholderia xenovorans LB400 & 2010 & Furfural & - Burkholderiales & Koopman et al. (2010b) \\
\hline Ralstonia eutropha & 2008 & Furfural & - Burkholderiales & Yu and Stahl (2008) \\
\hline Pseudomonas putida & 2008 & Furfural & - Pseudomonales & Trifonova et al. (2008a) \\
\hline Serratia plymouthica & 2008 & Furfural & - Enterobacteriales & Trifonova et al. (2008a) \\
\hline Rhizobium radiobacter & 2008 & Furfural & - Rhizobiales & Trifonova et al. (2008a) \\
\hline Methylobacterium radiotolerans & 2008 & Furfural & - Rhizobiales & Trifonova et al. (2008a) \\
\hline Leifsonia xyli ssp. xyli & 2008 & Furfural & + Actinomycetales & Trifonova et al. (2008a) \\
\hline Agromyces aurantiacus & 2008 & Furfural & + Actinomycetales & Trifonova et al. (2008a) \\
\hline Coniochaeta ligniaria & 2008 & Furfural & F Coniochaetales & Trifonova et al. (2008a) \\
\hline Coniochaeta ligniaria $\mathrm{c} 8$ & 2004 & Furfural and HMF & F Coniochaetales & Lopez et al. (2004) \\
\hline Methylobacterium extorquens & 2004 & Furfural and $\mathrm{HMF}^{\mathrm{b}}$ & - Rhizobiales & Lopez et al. (2004) \\
\hline Pseudomonas sp. & 2004 & $\mathrm{HMF}^{\mathrm{a}}$ & - Pseudomonadales & Lopez et al. (2004) \\
\hline Acinetobacter-like & 2004 & Furfural $^{\mathrm{a}}$ & - Pseudomonadales & Lopez et al. (2004) \\
\hline Flavobacterium indologenes & 2004 & Furfural and $\mathrm{HMF}^{\mathrm{b}}$ & - Flavobacteriales & Lopez et al. (2004) \\
\hline Stenotrophomonas maltophylia & 2004 & Furfural and $\mathrm{HMF}^{\mathrm{b}}$ & - Xanthomonadales & Lopez et al. (2004) \\
\hline Desulfovibrio $\mathrm{sp}$. & 1991 & Furfural $^{\mathrm{c}}$ & - Desulfovibrionales & Boopathy and Daniels (1991) \\
\hline Pseudomonas putida Fu-1 & 1989 & Furfural & - Pseudomonadales & Koenig and Andreesen (1989) \\
\hline Escherichia coli K-12 NAR30, NAR40 & 1986 & 2-Furoic acid & - Enterobacteriales & Abdulrashid and Clark (1987) \\
\hline Desufovibrio sp. strain $\mathrm{f}-1$ & 1983 & Furfural $^{\mathrm{c}}$ & - Desulfovibrionales & Brune et al. (1983) \\
\hline Pseudomonas putida $\mathrm{F} 2$ & 1969 & 2-Furoic acid & - Pseudomonadales & Trudgill (1969) \\
\hline Pseudomonas sp. 5863 & 1964 & 2-Furoic acid & - Pseudomonadales & Kakinuma and Yamatodani (1964) \\
\hline
\end{tabular}

a - Gram negative, + Gram positive, $F$ fungus

${ }^{\mathrm{b}}$ Based solely on substrate depletion

${ }^{\mathrm{c}}$ Anaerobic

These authors isolated "numerous microorganisms" capable of utilizing furanic compounds as a carbon source, several of which formed glutamic acid from 2-furoic acid. Since this early report, a few dozen other furan-degrading microorganisms have been identified, the majority of which very recently (Table 1).

Most furan-degrading microorganisms have been isolated using classical enrichment on HMF and/or furfural as a carbon source (Abdulrashid and Clark 1987; Boopathy et al. 1993; Koenig and Andreesen 1989; Lopez et al. 2004; Trudgill 1969; Wierckx et al. 2010; Yu and Stahl 2008). Trifonova and coworkers (Trifonova et al. 2008a, b) identified several furfural degraders in an attempt to isolate microorganisms that reduced the phytotoxicity of torrefied grass fibers, using an elegant assay based on the germination of lettuce seeds. The overview presented in Table 1 shows that most furanic compound-degrading microorganisms characterized thus far are aerobic Gram-negative bacteria, belonging to a relatively small number of genera. This suggests that furan catabolism can only be found among specialized microorganisms. It should be noted, however, that the list is strongly biased by our recent results (Koopman et al. 2010b), where we 
identified various new furan-degrading bacteria based on the presence of genes encoding homologues of the furanic catabolic enzymes of Cupriavidus basilensis HMF14.

Remarkably, only three fungal furanic aldehyde degraders have been reported to date, two Coniochaeta ligniaria strains (Trifonova et al. 2008a; Lopez et al. 2004) and Amorphotheca resinae ZN1 (Zhang et al. 2010). This underrepresentation of eukaryotes may be caused by the fact that fast-growing bacteria are more likely to emerge from short-term enrichment cultures. Alternative experimental setups such as the long-term enrichment used by Zhang et al. (2010) may lead to the identification of a greater number of furanic aldehyde-degrading fungi. Reports on anaerobic degradation of furfural are equally scarce: only two microorganisms, both Desulfovibrio strains (Brune et al. 1983; Boopathy and Daniels 1991), are known to convert furfural anaerobically, producing acetic acid. Similarly, until recently very few reports existed on microorganisms capable of degrading HMF. This is likely to be attributable to the focus on furfural degradation, in agreement with the abundance of this furanic compound in nature and with its widespread utilization in industry.

\section{Toxic effects of furanic compounds}

Aldehydes in general are highly reactive molecules, giving rise to the formation of reactive oxygen species (ROS; Feron et al. 1991; Zaldivar et al. 1999). Furanic aldehydes are no exception in this respect, causing ROS-associated damage to proteins, nucleic acids, and cell organelles (Almeida et al. 2009; Zaldivar et al. 1999; Allen et al. 2010). In furanic aldehyde-challenged Saccharomyces cerevisiae, expression of genes related to general stress adaptation and tolerance, central metabolism, transport, and degradation of damaged proteins were upregulated, indicating a system-wide stress response (Lin et al. 2009; Ma and Liu 2010). In addition to ROS-induced oxidative damage, furanic aldehydes exert several specific effects such as inhibition of enzymes in primary metabolism Modig et al. 2002). This typically leads to a heavily increased lag phase (Almeida et al. 2009; Mills et al. 2009) that may be overcome only by considerably increasing the inoculum density (Heer and Sauer 2008; Yu and Stahl 2008; Roberto et al. 1996). Furthermore, many microorganisms reduce or oxidize furanic aldehydes to their alcohol, respectively, carboxylic acid forms to ameliorate their toxic effect (Almeida et al. 2007, 2009; Wierckx et al. 2010). The toxic aldehyde can be eliminated in this way, but the cofactors required for these conversions (NAD/ $\mathrm{NADH}$ ) may be depleted, again increasing the lag phase (Lin et al. 2009; Liu 2006; Almeida et al. 2007).

\section{Biodegradation of furanic compounds}

As outlined above, reduction or oxidation of furanic aldehydes is quite commonly observed, and similar defense mechanisms against all sorts of aldehydes are found throughout nature. The oxidation or reduction of furanic aldehydes should not be confused, however, with degradation. Often, it is unclear to which extent furanic compounds are truly metabolized. In several reports, it is merely established that the furanic aldehydes have disappeared, without mention of the metabolic fate of the corresponding alcohols or carboxylic acids. This gives rise to much confusion in literature regarding the metabolic capacity of furanic compound "degrading" microorganisms. Therefore, all the different forms of the furanic compound (alcohol, aldehyde, and carboxylic acid) should be carefully monitored in order to establish whether the furanic aldehydes are actually metabolized or only transformed into a less toxic form (Wierckx et al. 2010).

The first biochemical route for aerobic furfural degradation was proposed by P.W. Trudgill in 1969, for the furfural-degrading bacterium Pseudomonas putida F2. This pathway was later verified and amended by Koenig and Andreesen (1990) and Koopman et al. (2010b). In the "Trudgill pathway" (Fig. 1b), furfural is first oxidized to 2-furoic acid by an aldehyde dehydrogenase (Koenig and Andreesen 1990). 2-Furoic acid is subsequently ligated to coenzyme-A by a furoyl-CoA synthetase, after which furoyl-CoA is hydroxylated at the $\mathrm{C}_{5}$ position by a furoyl-CoA dehydrogenase. The resulting enol-CoA tautomerizes to its keto form, which has a lactone ring structure. The lactone ring opens through (spontaneous or catalyzed) hydrolysis, and after another keto-enol tautomerization, 2-oxoglutaroyl-CoA is formed. Through hydrolysis of the CoA thioester 2-oxoglutarate is released, which is metabolized via the tricarboxylic acid cycle.

In 1989, Koenig and Andreesen demonstrated the validity of parts of this pathway at the enzyme level in $P$. putida Ful (Koenig and Andreesen 1989). The furoyl-CoA dehydrogenase was shown to be dependent on molybdenum, by labeling with the molybdenum antagonist $\left[{ }^{185} \mathrm{~W}\right]$ tungstate while molybdate was present in the media. The formation of 2-oxoglutarate as the end product of this degradation pathway was confirmed by the addition of arsenite, which specifically inhibits 2-oxoglutarate dehydrogenase (Koenig and Andreesen 1990; Koopman et al. 2010b).

Recently, we have demonstrated that $C$. basilensis HMF14 utilizes the Trudgill pathway for 2-furoic acid metabolism. Moreover, we were able to fully characterize this pathway at the genetic level (see below; Koopman et al. 2010b). In addition to the enzyme activities characterized 


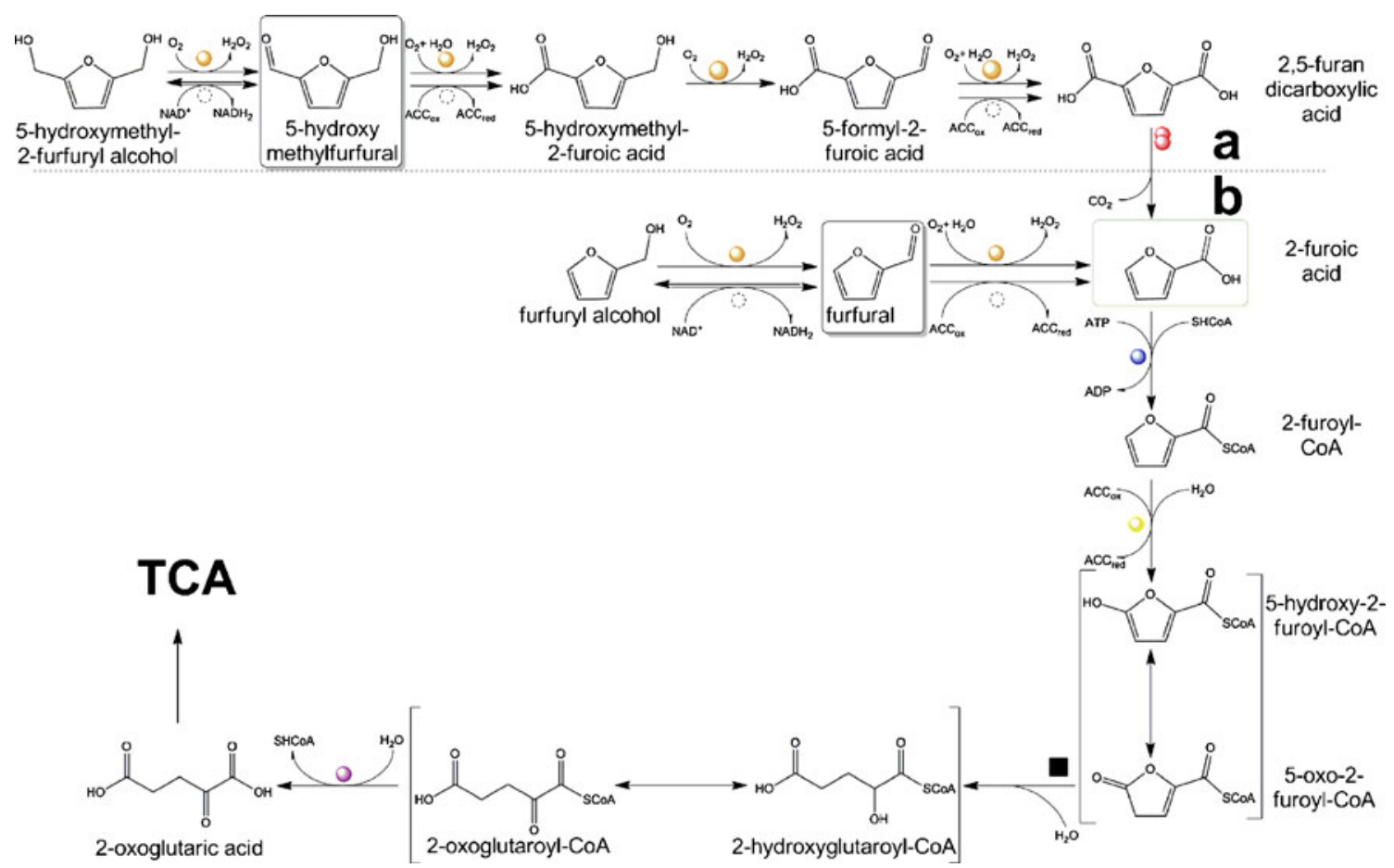

Fig. 1 Metabolic pathways of 5-hydroxymethylfurfural (a) and furfural (b) degradation in Cupriavidus basilensis HMF14, adapted from Koopman et al. (2010b) and Trudgill (1969). Colored symbols indicate enzymes with the following activities: orange furfural/HMF oxidoreductase, red 2,5-furandicarboxylic acid decarboxylase, blue 2furoyl-CoA synthetase, yellow furoyl-CoA dehydrogenase, purple 2-
oxoglutaryl-CoA hydrolase. Dashed circles indicate aspecific aldehyde or alcohol dehydrogenases. The black square indicates a lactone hydrolysis. Bracketed reactions indicate keto-enol tautomerizations. $A C C$ indicates acceptor, which is oxidized (ox) or reduced (red). TCA indicates the tricarboxylic acid cycle by Koenig and Andreesen (1990) and Trudgill (1969), one new enzyme was identified, i.e., a CoA thioesterase. Although CoA thioester hydrolysis had been included in the pathways proposed by both Trudgill and Koenig, it was left open whether or not an enzyme was involved. The CoA thioesterase of $C$. basilensis HMF14 was shown to be an essential enzyme for 2-furoic acid degradation. However, it was not clear whether the CoA thioester was either hydrolyzed in the final step leading to 2-oxoglutarate, or further upstream in the pathway. Additional biochemical characterization of this enzyme is needed to clarify its exact position in the pathway.

The genetic analysis suggested that furoyl-CoA dehydrogenase is indeed a molybdenum-dependent enzyme as demonstrated by Koenig and Andreesen (1989). However, contrary to the results obtained for $P$. putida Fu1, tungstate did not inhibit growth in $C$. basilensis HMF14 (unpublished data). This may well relate to the heavy metal resistance of C. basilensis, preventing tungstate from entering the cell (Goris et al. 2001).

In addition to 2-furoic acid degradation via the Trudgill pathway in $C$. basilensis HMF14, we also elucidated the HMF degradation pathway (Fig. 1a; Koopman et al. 2010 b). In analogy to furfural, HMF is first oxidized to the corresponding monocarboxylic acid, which is further oxidized to 2,5-furandicarboxylic acid (FDCA) by the specific oxidoreductase $\mathrm{HmfH}$. This latter transformation actually entails two oxidations, and based on the mechanism of other GMC-superfamily oxidoreductases, 5-formyl-2furoic acid may be expected as an intermediate product. So far, however, this compound has not been observed in the supernatant of $C$. basilensis HMF14 cultures on HMF. FDCA is subsequently decarboxylated to 2-furoic acid, after which the degradation proceeds via the Trudgill pathway. Thus, the HMF and furfural degradation pathways converge at the level of 2-furoic acid.

It should be noted that the furfural and HMF degradation routes are, in fact, 2-furoic acid and 5-hydroxymethyl-2furoic acid degradation routes. No specific aldehyde dehydrogenases were identified among the enzymes that are essential for furanic aldehyde catabolism, although the oxidoreductase of the HMF degradation pathway was shown to also oxidize the alcohol and aldehyde forms of both furfural and HMF (Koopman et al. 2010b). The enzymes of the "upper degradation pathways," comprising the initial furanic aldehyde and alcohol oxidations, are likely to be generic, broad-specificity dehydrogenases that defend the cells against toxic aldehydes. Apparently, the 
(5-hydroxymethyl-)2-furoic acid degradation "modules" have been recruited later in evolutionary history, constituting complete degradation pathways for furfural and HMF by complementing existing common aldehyde detoxification routes.

\section{Genetic background of furanic degradation routes}

Identification of genes involved in furanic aldehyde degradation

Although the degradation pathway for furfural was partially characterized at the enzyme level in the late 1980s, the genetic background has only recently been elucidated. Nichols and Mertens (2008) provided some preliminary insights by analysis of $P$. putida Ful transposon mutants that were no longer able to grow on 2-furoic acid. Two mutants were obtained in which either $p s f B$ or $p s f F$ was disrupted. The $p s f B$ gene encodes a lysR-type transcriptional regulator and is surrounded by the $p s f 2$ gene cluster. This cluster contains several genes, encoding a.o., an aldehyde dehydrogenase, and a xanthine dehydrogenase accessory factor that were proposed to have a role in furfural metabolism. However, a complete metabolic route could not be reconstructed. The $p s f F$ gene encodes a GcvRfamily transcriptional regulator and is surrounded by the psf 9 gene cluster, which is involved in general stress tolerance (Nichols and Mertens 2008).

By a similar approach of transposon mutant screening and nucleotide sequence analysis, we identified the two gene clusters in C. basilensis HMF14 that are responsible for the degradation of 2-furoic acid and the conversion of HMF to 2-furoic acid (Koopman et al. 2010b). The "furfural cluster" contains the hmfABCDE genes, encoding all the enzyme activities proposed by Trudgill (1969) and Koenig and Andreesen (1990) as well as the newly identified CoA thioesterase. The $h m f F G H$ gene cluster is involved in the metabolism of HMF to 2-furoic acid, via the key intermediate FDCA, as described in the previous paragraph. The ability to utilize HMF and furfural could be transferred to $P$. putida S12 by introducing the eight genes of the HMF and furfural clusters, confirming the functionality of the encoded enzymes. Furthermore, this unequivocally demonstrated that all essential genes for the metabolism of HMF and furfural had been identified (Koopman et al. 2010b).

Genetic organization of the genes involved in furanic compounds degradation

The availability of the genetic information included in the $h m f$ clusters allowed the identification of various other bacteria capable of degrading furfural and/or HMF (Fig. 2; Koopman et al. 2010b). When comparing the genetic organization of the $h m f$ genes in these bacteria, the order of the $h m f A B C D E$ genes is highly conserved.

The sequence similarity levels of individual $h m f A B C D E$ homologues in confirmed furfural degraders suggested a rather relaxed sequence-functionality relationship. Among the furfural degraders, the lowest homology to HmfA, the major subunit of the putative furoyl-CoA dehydrogenase, was observed for Dinoroseobacter shibae DFL12 (54\% identity). Apparently, even a moderate sequence similarity combined with the proximity of genes encoding a CoA synthetase, a molybdenum-dependent dehydrogenase and a CoA thioesterase can provide a good prediction of the furanic aldehyde-degrading capacities of strains previously not associated with this trait. Based on this observation, we extended the homology search of Koopman et al. (2010b) using somewhat less stringent search criteria. Figure 2 shows an updated and extended overview of the homology search, which now includes several Gram-positive bacteria. Among these, preliminary results indicate that Geobacillus kaustophilus HTA426 (Takami et al. 2004) can degrade furfural (unpublished data). In some of these Gram-positive putative furfural degraders, the structural order of the $h m f A B C D E$ homologues was different from the previously identified bacteria. Furthermore, more genes encoding enzymes with putative activities related to the furoyl-CoA dehydrogenase were found. It is known, however, that the domain organization of this type of molybdenum-dependent dehydrogenases can be quite variable (Kisker et al. 1998). Therefore, an alternative genetic organization does not necessarily imply a different catalytic activity, although the 2-furoic acid degradation capacities of the identified Gram positives remain to be confirmed.

The $h m f F G H$ cluster is much less conserved than the hmfABCDE cluster, both with regard to its spatial organization and with regard to homology of the encoded amino acid sequences (Koopman et al. 2010b). Some Burkholderia and Methylobacterium strains have all hmf genes organized in a single large cluster. However, in most (putative) HMF degraders, the $h m f F G$ and $h m f H$ genes are organized in a diverse manner. The hmfABCDE genes encode the core 2-furoic acid pathway, which is also required for HMF degradation. Therefore, a high level of conservation may be expected for these genes, whereas the genes for HMF degradation may have been recruited later from other, possibly diverse, sources.

Additional genes related to furanic aldehyde degradation or tolerance

In addition to the genes encoding the enzymes that constitute the furfural and HMF metabolic pathways, 
Ralstonia (Cupriavidus) basilensis HMF14 $\mathrm{HF}$

eutropha $\mathrm{H} 16^{\mathrm{F}}$

sp. 5_7_47FAA

eutropha JMP134

pickettii 12D

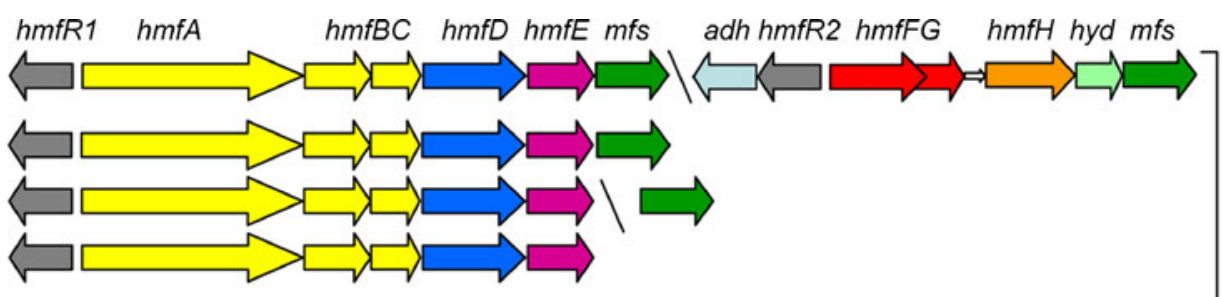

Burkholderia

phytofirmans PsJN ${ }^{H F}$ phymatum STM815 $\mathrm{HF}$ sp. CCGE1001/3

sp. CCGE1002 graminis C4D1M xenovorans LB400 $F$ sp. $\mathrm{CH} 1-1$

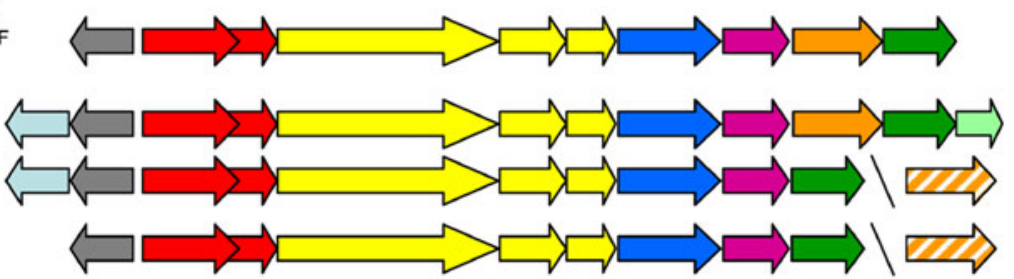

$\beta$-proteobacteria

Methylobacterium radiotolerans $\mathrm{JCM} 2831^{\mathrm{HF}}$

sp. $4-46$

nodulans ORS2060

Bradyrhizobium japonicum USDA110 $\mathrm{HF}$

Rhodopseudomonas palustris $\mathrm{BisB18} F$

Azospirilium sp. B510

Afipia

sp. $1 \mathrm{NLS} 2$
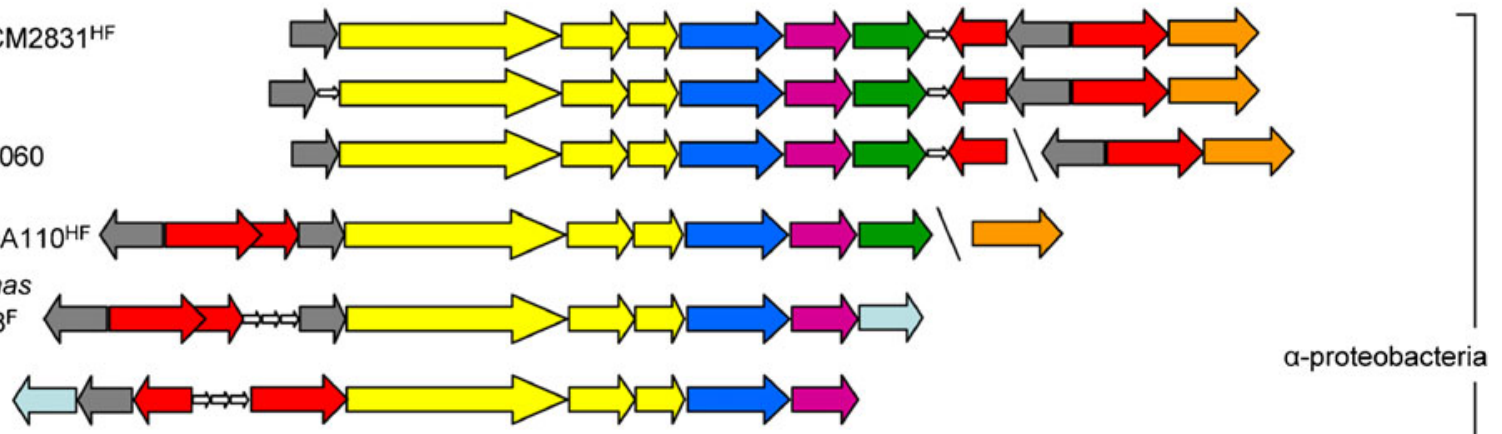

Acidiphilium

cryptum JF-5

multivorum AIU301

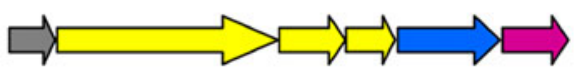

Dinoroseobacter shibae DFL12F

Geobacillus sp. Y4.1MC1

kaustophilus HTA426

Streptomyces violaceusniger TU4113

Pseudonocardia sp. P1

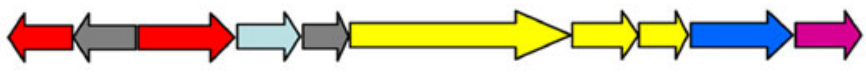

a-proteobacteria
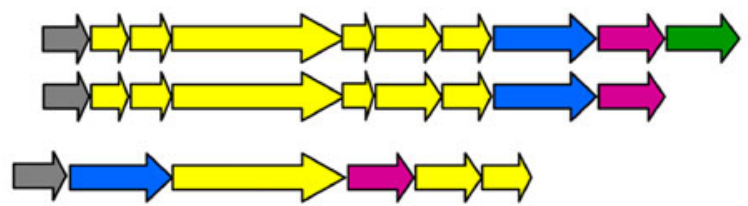

Gram+

Fig. 2 Spatial organization of the furfural and HMF gene clusters of potential and established furfural and/or HMF degrading bacteria. Adapted from Koopman et al. (2010b). Species were included in the list based on the presence of genes encoding homologues of HmfA, $\mathrm{HmfD}$ and $\mathrm{HmfE}$, with at least $40 \%$ similarity to the corresponding protein of $C$. basilensis HMF14, in each others' immediate vicinity. Gram-negative bacteria are subdivided into $\alpha$ - and $\beta$-proteobacteria. The following colors indicate putative encoded enzyme activities: gray transcriptional regulator, yellow furoyl-CoA dehydrogenase, blue
furoyl-CoA synthetase, purple oxoglutaryl-CoA thioesterase, green major facilitator superfamily transporter, light blue aldehyde dehydrogenase, red 2,5-furandicarboxylic acid decarboxylase, light green hydroxylase, orange $\mathrm{HMF} /$ furfural oxidoreductase, white arrows indicate genes with no relation to furfural or HMF metabolism. Superscript letters indicate strains which have been confirmed to degrade furfural $\left({ }^{F}\right)$ or both HMF and furfural $\left({ }^{H F}\right)$. Dashed orange arrows indicate putative $\mathrm{HMF} /$ furfural oxidoreductases which group into cluster 2 of Fig. 3 several additional genes have been identified that may be associated with furaldehyde degradation or tolerance. In close proximity to the $h m f$ genes of $C$. basilensis HMF14 are the $m f s, h m f R, a d h$, and hyd genes. Homologues of these genes can also be found in proximity to the $h m f$ genes of the various species depicted in Fig. 2. These genes are not essential for the degradation of HMF or furfural, but may play a role in transport, transcriptional regulation, and possibly redundant metabolic functions.

The latter may be true for the homologues of the $a d h$ gene of $C$. basilensis HMF14 (Fig. 2, light blue) that are found in the vicinity of the hmf genes in several species. 
The putative aldehyde dehydrogenases encoded by these genes show 59-69\% identity to the PsfA aldehyde dehydrogenase of $P$. putida Ful (Nichols and Mertens 2008). These genes may well contribute to the oxidation of furfural and/or HMF. Several genes have also been identified in $S$. cerevisiae and Escherichia coli that are responsible for the reduction of furanic aldehydes (Petersson et al. 2006; Gutiérrez et al. 2006)

In the mutant analyses of furanic compound-degrading microorganisms, several additional genes with similar functions were identified (Koopman et al. 2010b; Nichols and Mertens 2008). Examples are genes encoding peroxiredoxins and proteases that may be related to a general aldehyde-induced stress response (Zaldivar et al. 1999). Such genes may prove to be interesting targets for increasing the furanic aldehyde tolerance of industrial production hosts such as $S$. cerevisiae or E. coli.

Phylogeny of putative HMF/furfural oxidoreductase enzymes

Although the HMF metabolic pathway consists of only two specific activities, the $\mathrm{HmfH}$ enzyme seems to be the determining factor for HMF degradation. Several strains which are incapable of HMF degradation possess homologues of $h m f F G$. Vice versa, strains that are incapable of HMF degradation never possess a functional homologue of $h m f H$. Therefore, the presence of an $h m f H$ homologue seems to be representative for the capacity to degrade HMF. Burkholderia xenovorans LB400 appears to be an exception, since it apparently contains both $h m f H$ and $h m f F G$, but is unable to degrade HMF. Therefore, different $\mathrm{HmfH}$ homologues were analyzed in further detail by ClustalW2 cluster analysis. From this analysis, it is evident that two distinct clusters can be discriminated (Fig. 3).

Cluster 1 contains the $\mathrm{HmfH}$ homologues of established HMF-degrading bacteria, in addition to several bacteria whose HMF-degrading capacity has not yet been confirmed. The putative $\mathrm{HmfH}$ from B. xenovorans LB400, on the other hand, belongs to Cluster 2. Although this strain is the only confirmed non-HMF degrader, Cluster 2 also includes $\mathrm{HmfH}$ homologues of species that do not even possess significantly similar HmfABCDE homologues. As these are a prerequisite for HMF degradation, it appears likely that Cluster-2 $\mathrm{HmfH}$ homologues are not associated with the ability to degrade HMF. However, the correlation between the clustering behaviour of $\mathrm{HmfH}$ homologues and the (in-)ability to utilize HMF should be further confirmed.

It may be noted that some Burkholderia strains contain both a Cluster-1 and a Cluster-2 HmfH homologue. Presumably, the Cluster-1 $\mathrm{HmfH}$ is responsible for its ability to utilize HMF, since genes encoding this Cluster-1 $\mathrm{HmfH}$ are all in close proximity to the rest of the $h m f$ genes (Koopman et al. 2010b). The $\mathrm{HmfH}$ homologue of Bradyrhizobium japonicum USDA 110 presents an exceptional case. This strain does degrade HMF, but its $\mathrm{HmfH}$
Fig. 3 Cluster analysis of putative HMF/furfural oxidoreductases. The neighbor-joining plot was generated by TreeView (Page 1996) from a ClustalW2 alignment (Thompson et al. 2002) of all known proteins having at least $40 \%$ identity to $\mathrm{HmfH}$ from C. basilensis HMF14. The scale bar represents 0.1 substitutions per nucleotide position. Positive symbol behind the protein name indicates that this protein has been implicated in HMF degradation. Negative symbol indicates a protein in a species able to degraded furfural, but not HMF (Koopman et al. 2010b)

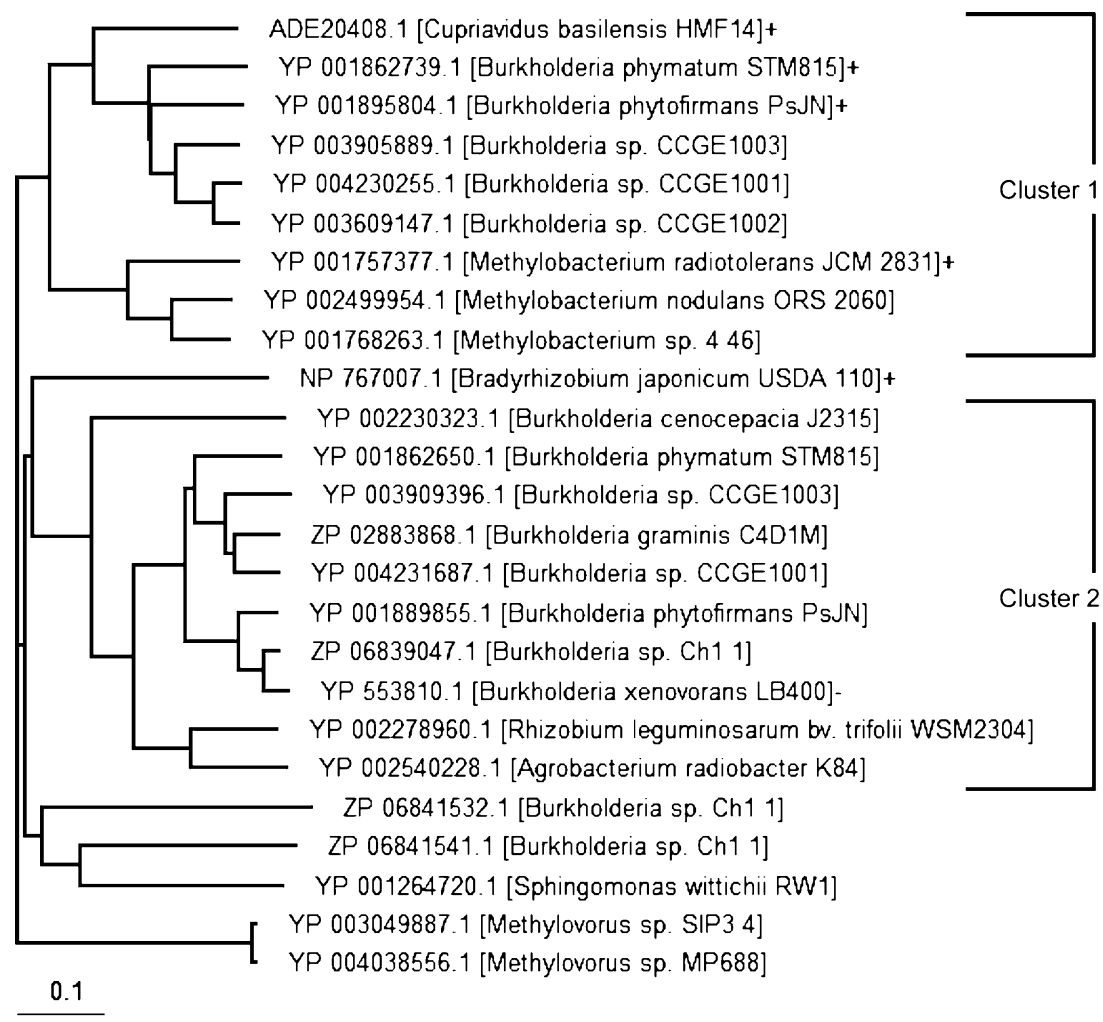


homologue does not fall within Cluster 1. However, clustering with Cluster 2 is also poor, suggesting this enzyme may have to be considered an atypical example of an HMF oxidoreductase.

\section{Conclusions: impact and future prospects}

\section{Biological detoxification}

The study of the microbial metabolism of furanic aldehydes has led to a number of new possibilities, mostly related to the use of lignocellulosic hydrolysates. Most studies performed to date have focused on the removal of furanic inhibitors from lignocellulosic hydrolysate using microorganisms that metabolize furfural and/or HMF (Nichols et al. 2008, 2010; Okuda et al. 2008; Wierckx et al. 2010; Parawira and Tekere 2011). Although this process of bioabatement holds great promise for increasing the fermentability of lignocellulosic hydrolysate, several drawbacks exist related to the types of microorganisms used. Most bioabatement processes described to date are performed aerobically at near-neutral $\mathrm{pH}$. Aerobic detoxification may be compatible with, or even be beneficial for, subsequent anaerobic fermentation. However, the high amount of energy required for aeration of hydrolysates (which often have a high solids content), and the risk of infection at near neutral $\mathrm{pH}$, pose a serious threat to the economic feasibility at an industrial scale. Additionally, most bioabatement organisms described also consume the sugar fraction which is highly undesirable unless the bioabatement organism is also the production host, as described by Yu and Stahl (2008).

A truly efficient bioabatement process would require an organism that consumes only inhibitors such as furanic aldehydes, preferably at low $\mathrm{pH}$ and under anaerobic conditions. Since such organisms are not available, these must either be retrieved from nature or engineered by implementing a furanic aldehyde degradation pathway in an anaerobic acidophile. Obviously, such a pathway may also be implemented directly in an industrial production host to enable simultaneous detoxification and fermentation. This approach would perfectly match a consolidated bioprocess setup, uniting not only biomass hydrolysis and fermentation in a single microorganism, but also removal of inhibitors that emerged from biomass pretreatment.

Opportunities and limitations for heterologous expression of furanic aldehyde catabolic pathways

As described above, we have functionally expressed the furfural and HMF pathways of $C$. basilensis HMF14 in $P$. putida S12 (Koopman et al. 2010b). This demonstrated that the furanic aldehyde pathways can-in principle-be applied in a heterologous host. By this approach, the efficiency of utilizing lignocellulosic hydrolysate as a biotechnological feedstock may be improved, since it would allow the microbial host to metabolize the toxic furanic aldehyde inhibitors.

It should be noted, however, that the specific properties of certain enzymes in the pathways pose limitations to their applicability. Based on homology to other enzymes, it is expected that the furoyl-CoA dehydrogenase encoded by $h m f A B C$ requires a specific molybdopterin cofactor (MoCo), the exact type of which is presently unclear. This limits the applicability of the furaldehyde catabolic pathways to hosts that possess the correct molybdopterin biosynthesis pathway. Although MoCos are widespread cofactors, the major industrial host $S$. cerevisiae lacks a molybdopterin biosynthesis pathway (Unkles et al. 1999). Implementation of the furanic aldehyde pathway in this host would therefore require the additional engineering of MoCo biosynthesis, which is a complex trait involving a large number of genes (Schwarz 2005). Furthermore, the oxygen dependency of the HMF/furfural oxidoreductase limits its application to aerobic processes, whereas most industrial fermentation processes are performed under micro-aerobic conditions at best.

\section{Production of FDCA}

As described above, oxygen dependency of the HMF/ furfural oxidoreductase limits its applicability in anaerobic detoxification. However, this unique enzyme may find industrial application for the production of FDCA. FDCA has been proclaimed by the US Department of Energy as one of a list of 12 promising biobased chemicals that should contribute to the "greening" of the chemical industry in the near future (Werpy and Peterson 2004). Through expression of the $C$. basilensis oxidoreductase in solvent-tolerant $P$. putida S12, we were able to obtain $30 \mathrm{~g} / \mathrm{l}$ of FDCA from HMF at a yield of $97 \%$ (Koopman et al. 2010a). FDCA is regarded as a biobased alternative to terephthalic acid for the production of polyethyleneterephthalate and other aromatics containing polymers, as well as an important platform compound for the synthesis of various chemical building blocks (Werpy and Peterson 2004; Bozell and Petersen 2010). As such, FDCA promises to be of great economic, as well as environmental benefit.

\section{Future prospects}

So far, the furfural and HMF degradation pathways of $C$. basilensis HMF14 constitute the only fully elucidated metabolic routes for these compounds (Koenig and Andreesen 1990; Koopman et al. 2010b; Trudgill 1969). 
It can obviously not be excluded that furanic compounds can be degraded via alternative routes. The observation that mostly relatively similar bacteria were identified as furan degraders based on the genetic information of the hmf genes of $C$. basilensis HMF14, as opposed to other established furan degraders, supports the latter view. It is therefore expected that the characterization of the metabolism of furanic compounds in fungi or anaerobic bacteria will lead to the discovery of alternative metabolic pathways. These will certainly make useful additions to the existing toolbox of furaldehyde degradation pathways that may not only help to overcome the limitations of the $C$. basilensis HMF14 pathway for industrial application, but also provide new opportunities for the biocatalytic production of furanic compound-based fine chemicals and building blocks.

Acknowledgements The authors would like to thank Dr. Hideto Takami of the Japan Agency for Marine-Earth Science \& Technology for providing a culture of Geobacillus kaustophilus HTA426.

Conflict of interest The authors declare that they have no conflict of interest.

Open Access This article is distributed under the terms of the Creative Commons Attribution Noncommercial License which permits any noncommercial use, distribution, and reproduction in any medium, provided the original author(s) and source are credited.

\section{References}

Abdulrashid N, Clark DP (1987) Isolation and genetic analysis of mutations allowing the degradation of furans and thiophenes by Escherichia coli. J Bacteriol 169(3):1267-1271

Abril D, Abril A (2009) Ethanol from lignocellulosic biomass. Cien Inv Agr 36(2):177-181

Allen SA, Clark W, McCaffery JM, Cai Z, Lanctot A, Slininger PJ, Liu ZL, Gorsich SW (2010) Furfural induces reactive oxygen species accumulation and cellular damage in Saccharomyces cerevisiae. Biotechnol Biofuels 3:2

Almeida JRM, Modig T, Petersson A, Hahn-Hagerdal B, Liden G, Gorwa-Grauslund MF (2007) Increased tolerance and conversion of inhibitors in lignocellulosic hydrolysates by Saccharomyces cerevisiae. J Chem Tech Biotechnol 82(4):340-349

Almeida JRM, Bertilsson M, Gorwa-Grauslund MF, Gorsich S, Liden G (2009) Metabolic effects of furaldehydes and impacts on biotechnological processes. Appl Microbiol Biotechnol 82 (4):625-638

Boopathy R, Daniels L (1991) Isolation and characterization of a furfural degrading sulfate-reducing bacterium from an anaerobic digester. Curr Microbiol 23(6):327-332

Boopathy R, Bokang H, Daniels L (1993) Biotransformation of furfural and 5-hydroxymethyl furfural by enteric bacteria. $\mathrm{J}$ Ind Microbiol Biotechnol 11:147-150

Bozell JJ, Petersen GR (2010) Technology development for the production of biobased products from biorefinery carbohydrates - the US Department of Energy's "Top 10" revisited. Green Chem 12(4):539-554
Brune G, Schoberth SM, Sahm H (1983) Growth of a strictly anaerobic bacterium on furfural (2-furaldehyde). Appl Environ Microbiol 46(5):1187-1192

Chheda JN, Roman-Leshkov Y, Dumesic JA (2007) Production of 5-hydroxymethylfurfural and furfural by dehydration of biomassderived mono- and poly-saccharides. Green Chem 9(4):342-350

Dean FM (1963) Naturally occurring oxygen ring compounds. Butterworths, Londen

Ding X, Wang MY, Yao YX, Li GY, Cai BC (2010) Protective effect of 5-hydroxymethylfurfural derived from processed Fructus Corni on human hepatocyte $\mathrm{LO} 2$ injured by hydrogen peroxide and its mechanism. J Ethnopharmacol 128(2):373-376

Feron VJ, Til HP, Devrijer F, Woutersen RA, Cassee FR, Vanbladeren PJ (1991) Aldehydes - occurrence, carcinogenic potential, mechanism of action and risk assessment. Mutat Res 259(3-4):363-385

Flaschenträger B, Wahhab SMA (1960) An improved method for the isolation and estimation of furan-2,5-dicarboxylic acid in human urine. Microchim Acta 48(2):275-281

Goris J, De Vos P, Coenye T, Hoste B, Janssens D, Brim H, Diels L, Mergeay M, Kersters K, Vandamme P (2001) Classification of metal-resistant bacteria from industrial biotopes as Ralstonia campinensis sp. nov., Ralstonia metallidurans sp. nov. and Ralstonia basilensis Steinle et al. 1998 emend. Int J Syst Evol Microbiol 51(Pt 5):1773-1782

Gutiérrez T, Ingram LO, Preston JF (2006) Purification and characterization of a furfural reductase (FFR) from Escherichia coli strain LYO1 - an enzyme important in the detoxification of furfural during ethanol production. J Biotechnol 121(2):154-164

Heer D, Sauer U (2008) Identification of furfural as a key toxin in lignocellulosic hydrolysates and evolution of a tolerant yeast strain. Microbial Biotechnol 1(6):497-506

Jing X, Zhang X, Bao J (2009) Inhibition performance of lignocellulose degradation products on industrial cellulase enzymes during cellulose hydrolysis. Appl Biochem Biotechnol 159(3):696-707

Kakinuma A, Yamatodani S (1964) L-Glutamic acid formation from 2-furoic acid by soil bacteria. Nature 201:420-421

Kawarada A, Takahashi N, Kitamura H, Seta Y, Takai M, Tamura S (1954) Biochemical studies on "Bakanae" fungus. Part 33. Bull Agr Chem Soc Japan 19:84-86

Kisker C, Schindelin H, Baas D, Retey J, Meckenstock RU, Kroneck PMH (1998) A structural comparison of molybdenum cofactorcontaining enzymes. FEMS Microbiol Rev 22(5):503-521

Klinke HB, Thomsen AB, Ahring BK (2004) Inhibition of ethanolproducing yeast and bacteria by degradation products produced during pre-treatment of biomass. Appl Microbiol Biotechnol 66 (1): $10-26$

Koenig K, Andreesen JR (1989) Molybdenum involvement in aerobic degradation of 2-furoic acid by Pseudomonas putida Fu1. Appl Environ Microbiol 55(7):1829-1834

Koenig K, Andreesen JR (1990) Xanthine dehydrogenase and 2-furoylcoenzyme A dehydrogenase from Pseudomonas putida Ful: two molybdenum-containing dehydrogenases of novel structural composition. J Bacteriol 172(10):5999-6009

Koopman F, Wierckx N, de Winde JH, Ruijssenaars HJ (2010a) Efficient whole-cell biotransformation of 5-(hydroxymethyl) furfural into FDCA, 2,5-furandicarboxylic acid. Bioresource Technol 101(16):6291-6296

Koopman F, Wierckx N, DeWinde JH, Ruijssenaars HJ (2010b) Identification and characterization of the furfural and 5(hydroxymethyl)furfural degradation pathways of Cupriavidus basilensis HMF 14. Proc Nat Acad Sci USA 107(11):4919-4924

Kumar P, Barrett DM, Delwiche MJ, Stroeve P (2009) Methods for pretreatment of lignocellulosic biomass for efficient hydrolysis and biofuel production. Ind Eng Chem Res 48(8):3713-3729

Larsson S, Palmqvist E, Hahn-Hagerdal B, Tengborg C, Stenberg K, Zacchi G, Nilvebrant NO (1999) The generation of fermentation 
inhibitors during dilute acid hydrolysis of softwood. Enzyme Microb Technol 24(3-4):151-159

Lin Y, Tanaka S (2006) Ethanol fermentation from biomass resources: current state and prospects. Appl Microbiol Biotechnol 69 (6):627-642

Lin AS, Qian K, Usami Y, Lin L, Itokawa H, Hsu C, Morris-Natschke SL, Lee KH (2008) 5-Hydroxymethyl-2-furfural, a clinical trials agent for sickle cell anemia, and its mono/di-glucosides from classically processed steamed rehmanniae radix. J Nat Med 62(2):164-167

Lin FM, Qiao B, Yuan YJ (2009) Comparative proteomic analysis of tolerance and adaptation of ethanologenic Saccharomyces cerevisiae to furfural, a lignocellulosic inhibitory compound. Appl Environ Microbiol 75(11):3765-3776

Liu ZL (2006) Genomic adaptation of ethanologenic yeast to biomass conversion inhibitors. Appl Microbiol Biotechnol 73(1):27-36

Lopez MJ, Nichols NN, Dien BS, Moreno J, Bothast RJ (2004) Isolation of microorganisms for biological detoxification of lignocellulosic hydrolysates. Appl Microbiol Biotechnol 64(1):125-131

Ma M, Liu ZL (2010) Comparative transcriptome profiling analyses during the lag phase uncover YAP1, PDR1, PDR3, RPN4, and HSF1 as key regulatory genes in genomic adaptation to the lignocellulose derived inhibitor HMF for Saccharomyces cerevisiae. BMC Genomics 11:660

Medina VG, Almering MJH, van Maris AJA, Pronk JT (2010) Elimination of glycerol production in anaerobic cultures of a Saccharomyces cerevisiae strain engineered to use acetic acid as an electron acceptor. Appl Environ Microbiol 76(1):190-195

Michail K, Matzi V, Maier A, Herwig R, Greilberger J, Juan H, Kunert O, Wintersteiger R (2007) Hydroxymethylfurfural: an enemy or a friendly xenobiotic? A bioanalytical approach. Anal Bioanal Chem 387(8):2801-2814

Mills TY, Sandoval NR, Gill RT (2009) Cellulosic hydrolysate toxicity and tolerance mechanisms in Escherichia coli. Biotechnol Biofuels 2:11

Modig T, Lidén G, Taherzadeh MJ (2002) Inhibition effects of furfural on alcohol dehydrogenase, aldehyde dehydrogenase and pyruvate dehydrogenase. Biochem J 363(3):769-776

Mussatto SI, Roberto IC (2004) Alternatives for detoxification of diluted-acid lignocellulosic hydrolyzates for use in fermentative processes: a review. Bioresource Technol 93(1):1-10

National Toxicology Program (1990) Toxicology and carcinogenesis studies of furfural (CAS No. 98-01-1) in F344/N rats and B6C3F1 mice (gavage studies). Natl Toxicol Program Tech Rep Ser 382:1-201

National Toxicology Program (2010) NTP toxicology and carcinogenesis studies of 5-(Hydroxymethyl)-2-furfural (CAS No. 67$47-0$ ) in $\mathrm{F} 344 / \mathrm{N}$ rats and B6C3F1 mice (gavage studies). Natl Toxicol Program Tech Rep Ser 554:7-13, 15-19, 21-31 passim

Nichols NN, Mertens JA (2008) Identification and transcriptional profiling of Pseudomonas putida genes involved in furoic acid metabolism. FEMS Microbiol Lett 284(1):52-57

Nichols NN, Sharma LN, Mowery RA, Chambliss CK, van Walsum GP, Dien BS, Iten LB (2008) Fungal metabolism of fermentation inhibitors present in corn stover dilute acid hydrolysate. Enzyme Microb Technol 42(7):624-630

Nichols NN, Dien BS, Cotta MA (2010) Fermentation of bioenergy crops into ethanol using biological abatement for removal of inhibitors. Bioresource Technol 101(19):7545-7550

Okuda N, Soneura M, Ninomiya K, Katakura Y, Shioya S (2008) Biological detoxification of waste house wood hydrolysate using Ureibacillus thermosphaericus for bioethanol production. J Biosci Bioeng 106(2):128-133

Page RDM (1996) TreeView: an application to display phylogenetic trees on personal computers. Comput Appl Biosci 12(4):357-358

Palmqvist E, Hahn-Hagerdal B (2000a) Fermentation of lignocellulosic hydrolysates. I: inhibition and detoxification. Bioresource Technol 74:17-24
Palmqvist E, Hahn-Hagerdal B (2000b) Fermentation of lignocellulosic hydrolysates. II: inhibitors and mechanisms of inhibition. Bioresource Technol 74:25-33

Parawira W, Tekere M (2011) Biotechnological strategies to overcome inhibitors in lignocellulose hydrolysates for ethanol production: review. Crit Rev Biotechnol 31(1):20-31

Petersson A, Almeida JRM, Modig T, Karhumaa K, Hahn-Hägerdal B, Gorwa-Grauslund MF, Lidén G (2006) A 5-hydroxymethyl furfural reducing enzyme encoded by the Saccharomyces cerevisiae ADH6 gene conveys HMF tolerance. Yeast 23:455-464

Ragauskas AJ, Williams CK, Davison BH, Britovsek G, Cairney J, Eckert CA, Frederick WJ, Hallett JP, Leak DJ, Liotta CL, Mielenz JR, Murphy R, Templer R, Tschaplinski T (2006) The path forward for biofuels and biomaterials. Science 311(5760):484- 489

Roberto IC, Sato S, deMancilha IM (1996) Effect of inoculum level on xylitol production from rice straw hemicellulose hydrolysate by Candida guilliermondii. J Ind Microbiol 16(6):348-350

Schwarz G (2005) Molybdenum cofactor biosynthesis and deficiency. Cell Mol Life Sci 62(23):2792-2810

Sumiki Y (1931) Studies on the fermentation products by mould fungi. Part IX. Bull Agr Chem Soc Japan 7:62-63

Taherzadeh MJ, Karimi K (2008) Pretreatment of lignocellulosic wastes to improve ethanol and biogas production: a review. Int $\mathrm{J}$ Mol Sci 9(9):1621-1651

Taherzadeh MJ, Eklund R, Gustafsson L, Niklasson C, Liden G (1997) Characterization and fermentation of dilute-acid hydrolysates from wood. Ind Eng Chem Res 36:4659-4665

Takami H, Takaki Y, Chee GJ, Nishi S, Shimamura S, Suzuki H, Matsui S, Uchiyama I (2004) Thermoadaptation trait revealed by the genome sequence of thermophilic Geobacillus kaustophilus. Nucleic Acids Res 32(21):6292-6303

Thompson JD, Gibson TJ, Higgins DG (2002) Multiple sequence alignment using ClustalW and ClustalX. Curr Protoc Bioinformatics Chapter 2:Unit 2.3

Thomsen MH, Thygesen A, Thomsen AB (2009) Identification and characterization of fermentation inhibitors formed during hydrothermal treatment and following SSF of wheat straw. Appl Microbiol Biotechnol 83(3):447-455

Trifonova R, Postma J, Ketelaars JJ, van Elsas JD (2008a) Thermally treated grass fibers as colonizable substrate for beneficial bacterial inoculum. FEMS Microbiol Ecol 56(3):561-571

Trifonova R, Postma J, Verstappen FW, Bouwmeester HJ, Ketelaars JJ, van Elsas JD (2008b) Removal of phytotoxic compounds from torrefied grass fibres by plant-beneficial microorganisms. FEMS Microbiol Ecol 66(1):158-166

Trudgill PW (1969) Metabolism of 2-Furoic Acid by Pseudomonas F2. Biochem J 113(4):577-587

Unkles SE, Heck IS, Appleyard M, Kinghorn JR (1999) Eukaryotic molybdopterin synthase-biochemical and molecular studies of Aspergillus nidulans cnxG and cnxH mutants. J Biol Chem 274 (27):19286-19293

Werpy T, Peterson G (2004) Top value added chemicals from biomass. Available at http://wwwostigov/bridge/

Wierckx N, Koopman F, Bandounas L, deWinde JH, Ruijssenaars HJ (2010) Isolation and characterization of Cupriavidus basilensis HMF14 for biological removal of inhibitors from lignocellulosic hydrolysate. Microbial biotechnol 3:336-343

Yu J, Stahl H (2008) Microbial utilization and biopolyester synthesis of bagasse hydrolysates. Bioresource Technol 99(17):8042-8048

Zaldivar J, Martinez A, Ingram LO (1999) Effect of selected aldehydes on the growth and fermentation of ethanologenic Escherichia coli. Biotechnol Bioeng 65(1):24-33

Zhang JA, Zhu ZN, Wang XF, Wang N, Wang W, Bao J (2010) Biodetoxification of toxins generated from lignocellulose pretreatment using a newly isolated fungus, Amorphotheca resinae $\mathrm{ZN1}$, and the consequent ethanol fermentation. Biotechnol Biofuels 3:15 